\title{
Seroprevalence of zoonotic diseases among farm animals in Kvemo Kartli (Georgia)
}

\author{
K Goginashvili¹, M donduashvili', Gaga Osiashvili', Ryan Arner² and Lile Malania ${ }^{3}$ \\ 'Laboratory of the Ministry of Agriculture, Tbilisi, Georgia; ${ }^{2}$ Metabiota Inc, San Fransisco, CA, USA; ${ }^{3}$ National Center for Disease \\ Control and Public Health, Tbilisi, Georgia
}

\section{Objective}

The purpose of this research was to study the seroprevalence of zoonotic diseases among farm animals in the Kvemo Kartli region of Georgia.

\section{Introduction}

Zoonotic diseases are an important cause of human morbidity and mortality; around $75 \%$ of recently emerging human infectious diseases are zoonoses. Herein we report the first seroprevalence study to include a range of emerging or re-emerging zoonotic pathogens of economic concern (including: Bacillus anthracis, Coxiella burnetii, Francisella spp., Brucella spp., and Crimean-Congo hemorrhagic fever virus (CCHFV)) affecting domestic animals (e.g., cattle, sheep, goat, and dog) in Georgia.

\section{Methods}

Cattle ( $\mathrm{n}=177)$ from Gardabani, Marneuli, and Tsalka (Kvemo Kartli region) were sampled for the study as were small ruminants and dogs $(\mathrm{n}=30)$. Bacillus anthracis, Brucella spp., CCHFV, and C. burnetii (Phase I) were detected using ELISA methods. Francisella tularensis was detected using a microscopic agglutination test (MAT).

\section{Results}

Of the cattle sampled, 11 were positive for $F$. tularensis, 39 were positive for Brucella spp., and seven were positive for C. burnetii. All samples were negative for CCHFV. Three goat samples were positive for C. burnetii, one goat sample and one dog sample were positive for F. tularensis.

\section{Conclusions}

Domestic animals serve as a source of disease that can spread to humans through vectors or direct contact. In Georgia, domestic animals were not previously studied for exposure to zoonotic diseases, with the exception of cattle, which were surveyed for brucellosis. In particular, the finding of $F$. tularensis seropositive animals is novel in Georgia, as this region was considered free of the pathogen. Screening studies of domestic/farm animals for zoonotic pathogens such as this can serve as a source of baseline data for regional risk assessments and to better inform One Health measures.

\section{Keywords}

Emerging diseases; Re-emerging diseases; Zoonotic diseases; Crimean-Congo hemorrhagic fever

\section{Acknowledgments}

The research study described in this presentation was made possible by financial support provided by the US Defense Threat Reduction Agency. The findings, opinions and views expressed herein belong to the authors and do not reflect an official position of the Department of the Army, Department of Defense, or the US Government, or any other organization listed

\section{${ }^{*}$ K Goginashvili}

E-mail: Goginashvili@Ima.ge 